\title{
Cost versus flexibility of different capacity leasing approaches on the optical network layer
}

\author{
Sofie Verbrugge, Didier Colle, Mario Pickavet, Piet Demeester \\ Dept. of Information Technology, Ghent University - IBBT, \\ Sint-Pietersnieuwstraat 41, B-9000 Gent, Belgium, \\ tel. +32 933 14900, fax +3293314899 \\ \{sofie.verbrugge, didier.colle, mario.pickavet, piet.demeester\} intec.ugent.be
}

\begin{abstract}
This paper discusses different capacity leasing scenarios for the optical network. It aims at providing systematic understanding of cost structure of optical networks, without focusing on technical details. It reviews different kinds of capacity leasing approaches discussing the relative costs and the flexibility associated to them to adapt to future traffic needs. Then it applies the Real Options principles to evaluate the most convenient planning solution. Real Option valuation is shown to be the formalization of the natural way of valuating different alternatives under uncertainty, taking into account that information becoming available during the course of the planning horizon might influence the strategy followed. The goal of the paper is to give insight in the practical applicability of the technique for the network operator.
\end{abstract}

Keywords: optical layer capacity, dark fiber, IRU, flexibility, Real Options

\section{Introduction}

The presence of multiple telecommunication providers and their past investments in optical layer capacity, have led to a wide availability of dark fiber throughout Europe. Therefore, for a network operator planning to deploy a new network, there is no need to acquire all physical capacity himself. Leasing capacity gives more flexibility and is therefore considered an attractive solution. A distinction can be made between traditional leasing and longer term Indefeasible Right of Use. Moreover, either dark fiber of wavelengths can be the subject of the lease.

This paper describes several capacity leasing scenarios, indicating typical costs and contract terms. We indicate useful approaches to evaluate the associated investment decisions from the perspective of the user of the lease and focus on the flexibility to switch between network scenarios and the relation between future traffic uncertainty and network flexibility, using Real Options thinking. 


\section{Capacity leasing approaches}

\subsection{IRU versus lease}

Leasing is a concept where the grantor grants the use of the asset to the grantee (user) for the duration of the lease, a well-known example is leasing a car where the asset considered is the car. In telecom, the lease of capacity on someone else's network can for example take the form of dark fiber or wavelength lease. A lease usually applies for a relatively short term, e.g. one to five years.

An Indefeasible Right of Use (IRU) is similar to a lease, but usually applies to a longer term, e.g. 15 or 20 years. For instance, assume operator $\mathrm{X}$ aggressively builds a worldwide fiber-optic network. If another operator $\mathrm{Y}$ is building a network but not in the same places as operator $\mathrm{X}$, to expand its reach, operator $\mathrm{Y}$ might buy an IRU for two fibers in operator X's network for 20 years [1].

\subsection{Dark fiber versus wavelength}

After the liberalization of the telecom market and the strong past investment in optical layer capacity, a lot of dark fiber is available. This means that, apart from the traditional deployment or acquisition of an optical layer network, a network operator nowadays also has the possibility to lease the optical layer capacity. He can deploy the higher layer network infrastructure based on this.

The form of the optical layer capacity the grantor of the lease offers to the grantee, can either be dark fiber or wavelengths. Both a dark fiber and a wavelength network are based on wavelength infrastructure in the network nodes. In a dark fiber network, the transmission infrastructure like multiplexers and transponders is owned by the grantee, whereas in a leased wavelength network this equipment is owned by the grantor of the lease. Several candidate node configurations can be distinguished, depending on the physical topology at hand and the requirement to have grooming capabilities (packing low capacity traffic in high capacity streams). More information on possible node configurations can be found in [2]. If we consider an optical ring network based on optical add-drop multiplexers (OADMs), on top of which DXCs and higher layer equipment is deployed, the costs for the optical network layer for both situations include:

- In case of a leased wavelength network: costs of the lease contracts

- In case of a dark fiber network: costs of the lease contracts, costs of the optical add-drop multiplexers (OADMs), costs of the transponders and costs of the WDM multiplexers

The costs of the DXCs and the other higher layer equipment will be similar in both cases. 


\subsection{Cost and flexibility}

As indicated above, IRUs usually have longer contract terms than ordinary leasing contracts. Furthermore, leasing of dark fiber also usually applies for longer terms than leasing of wavelengths. Fig. 1 compares IRU on dark fiber (IRU on DF) with a cost of 0,4 euro/meter/year, dark fiber lease (DF lease) with a cost of 1,2 euro/meter/year and wavelength lease (lambda lease) with a cost of 1,5 euro/meter/year. The mentioned link prices are in line with values found in the literature [3].

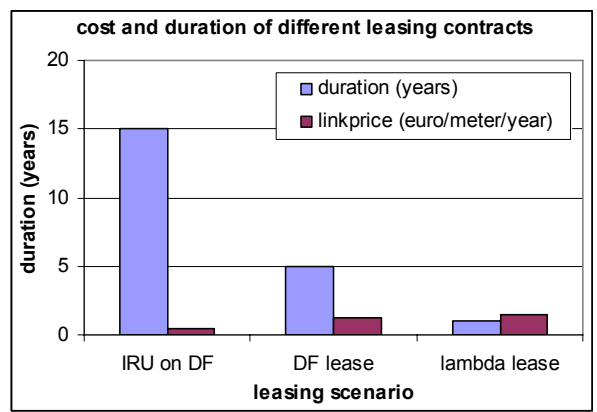

Fig. 1. Comparison of leasing contracts in term of costs

In order to make a full comparison of the optical layer costs of the different network deployment scenarios, also equipment $\operatorname{costs}^{1}$ for the transmission equipment are to be taken into account. Some reference costs can be found in Tab. 1 .

Tab. 1. Reference costs for optical layer equipment

\begin{tabular}{|l|l|l|l|}
\hline equipment & OADM & WDM mux (40 lambdas) & transponder 10G \\
\hline cost (euro) & 17000 & 37500 & 23000 \\
\hline
\end{tabular}

The duration of the contract has some relation with the flexibility: shorter contract terms lead to additional flexibility because the leased capacity can easily be increased or decreased at the end of the lease contract, e.g. by taking a lease for a higher number of wavelengths or by releasing a wavelength after one year in case of the lambda lease scenario. This type of flexibility is illustrated in Fig. 2, where the horizontal arrows represent the lease contracts (full lines for the IRU on DF, dashed lines for the DF lease and dotted lines for the lambda lease scenarios) and the vertical arrows indicate the possibility to increase or decrease the capacity at the end of the contract term by extending or releasing the leased capacity for the considered contract. Apart from the limited flexibility to increase or decrease capacity within a certain scenario (at the end of a contract term) indicated above, there is also the flexibility to switch between the considered scenarios at the end of the contract term, this means after 15 years for the

\footnotetext{
${ }^{1}$ The cost to set up the network and install this equipment is mainly labour costs and constitutes produced fixed assets, which are to be counted as CapEx together with the equipment itself. The labour costs for first-time network installation could be treated using an activity-based process description model [11], however, they are neglected here.
} 
IRU on DF, after 5 years for the DF lease and yearly for the leased wavelengths. Note that, in real life, there is also the possibility to prematurely end the contract, e.g. end the IRU before the end of the 15 year period by paying a kind of penalty fee, but this is not considered here.

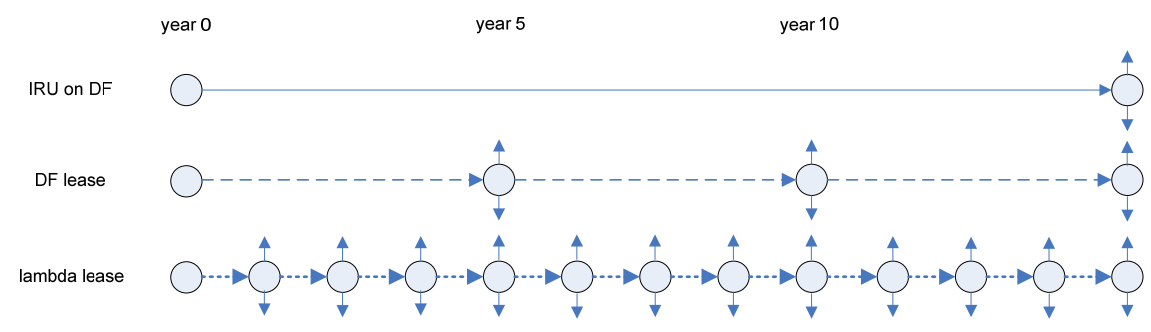

Fig. 2. Flexibility based on leasing contract duration

Given the fact the longer leasing contracts tend to be cheaper on a yearly basis than shorter ones, it may be beneficial to use IRU on DF in cases where the required capacity for the future years is expected to be non-decreasing. However, in cases where capacity decrease can be expected, shorter term contracts can be worthwhile, e.g. because of a competitor taking over some part of the market share in a particular area of the network where the considered operator is no longer interested in). Moreover, in an uncertain situation, shorter leasing contracts allow to follow a waitand-see approach. If the situation becomes clear after some time, it is possible to quickly react to it by releasing capacity or by moving to a leasing contract with a longer duration.

\section{Traffic uncertainty and how to cope with it}

\subsection{Uncertain traffic evolutions}

Unfortunately, there is no single, unbiased source of information on IP traffic growth. The National Science Foundation in the US stopped measuring the growth of the IP traffic on the Internet backbone in 1995. Nevertheless, some numbers can be found in literature. Growth rates till $150 \%, 200 \%$ or even more were often cited before the Internet bubble exploded [4]. Although overall IP traffic can be expected to continue growing, the actual growth rate is unclear. Moreover, when considering a particular network offering particular services the situation becomes even more cumbersome. Several forecasting methods are distinguished in the literature [5][6]. Based on accurate observations and the use of a suitable planning technique, several cases can be distinguished where part of the initial uncertainty disappears in the beginning of the planning horizon, so that more accurate planning becomes possible after that time, the so-called learning time. This can for example be the case when a 
competitor is entering the market but is unclear beforehand what market segment he will actually focus on. We will focus on these cases in the remainder of this paper.

\subsection{Flexibility to react to uncertain evolutions}

Based on additional information becoming available during the learning time in the first part of the planning horizon, the comparison of different possible network deployment scenarios might be very different from the initial expected situation. Consider the example of

Fig. 3. where we observe two possible network deployment paths, one consisting of a single path (no subpaths) and the other one containing two subpaths with two alternatives for the last subpath.

- When evaluating the single deployment path under traffic uncertainty, the only solution is to calculate its average cost over the different uncertain future evolutions. In case of a $60 \%$ chance of a traffic growth and a $40 \%$ chance of a status quo in the traffic, the expected cost for this deployment path would be

$$
0.6 * \operatorname{cost}_{\text {traffic growth }}(a)+0.4 * \operatorname{cost}_{\text {consta nt traffic }}(a)=E_{\text {uncertain traffic evolutions }}[\operatorname{cost}(a)]
$$

- When evaluating the path with the alternative subpaths, the expected cost for the first subpath (calculated in the same way as the path without flexibility above) will be augmented with the cost of the cheapest alternative for the second subpath. If the choice between alternative $c$ and $d$ is to be made at the beginning of the deployment path, there is no flexibility to react to changes happening during the first subpath. In that case the expected cost of the last subpath is to be calculated as the minimum value of the average expected costs for both alternative subpaths

$$
\min \left[E_{\text {uncertain traffic evolutions }}[\operatorname{cost}(c), \operatorname{cost}(d)]\right]
$$

- However, when the decision between $c$ and $d$ is only made at the end of subpath $b$, there is true flexibility, so that at that point in time the cheapest alternative will be chosen, taking into account the actual situation at the end of subpath $b$ (i.e. knowing possible evolutions that have taken place during the course of $b$ ). In that case the value of the second subpath is to be calculated as the average value over all expected traffic scenarios of this minimum cost

$$
E_{\text {uncertain traffic evolutions }}[\min [\operatorname{cost}(c), \operatorname{cost}(d)]]
$$

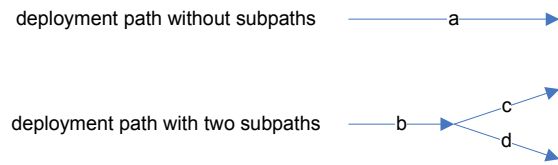

Fig. 3. Different deployment paths

\footnotetext{
${ }^{2}$ Remark that we use $\mathrm{E}[\mathrm{x}, \mathrm{y}]$ as a short notation for $\mathrm{E}[\mathrm{x}], \mathrm{E}[\mathrm{y}]$.
} 
The above observations indicate that the solution with the lowest cost based on the expected situation before the start of the deployment path (choice without flexibility) may be different from the solution with the lowest cost based on the knowledge after the first subpath (choice taking into account flexibility). When comparing the deployment paths $a$ (without flexibility) and the paths $b-c-d$ with the real flexibility to choose between $c$ and $d$ after the first subpath, the costs to be compared for the overall paths are

$$
E_{\text {uncertain traffic evolutions }}[\operatorname{cost}(a)]
$$

versus

$$
E_{\text {uncertain traffic evolutions }}[b+\min [\operatorname{cost}(c), \operatorname{cost}(d)]]
$$

\subsection{Real Options thinking}

Real Options thinking originates from the financial world. An option can be defined as the right for a limited time, to buy or sell the underlying value for a predetermined exercise price. Exercising the option (i.e. buying or selling the underlying value) is always optional; it is a right, not an obligation. This right holds for a predetermined time, till the so-called exercise date. The underlying value is the asset which the option concerns; this may be assets, real estate, precious metals, etc. The exercise price is the price for which the option can be exercised by its holder.

The term Real Options was introduced in 1977 [7]. It referred to the application of option pricing theory to the valuation of investments in real assets where a large part of the value is attributable to flexibility and learning over time. After some academic attention in the 1980s, interest in real options from industry rose considerably since mid 1990s. Real Options thinking has been successfully applied to determine the value of flexibility when deploying mines, manufacturing plants, etc. Application in the telecom business started recently [8][9], however, those papers do not aim at practical applicability of the methodology for the network operator. [10] provides a comprehensive introduction to Real Options theory, with a lot of practical examples. Real Options valuation can be seen as the formalization of the natural valuation for a deployment path with flexibility as described above. Referring back to

Fig. 3, the deployment path with flexibility gives us the options to choose between subpaths $c$ and $d$ later on, when choosing subpath $b$ in the beginning of the planning horizon. On the other hand, when taking the single deployment path $a$ immediately, there is no option later on, we need to stick to this path anyway. The path with the two alternative subpaths can be seen as holding an option to choose between them later on, when more information is available. The option price (price to obtain the option) in this case is the price difference between the subpath $b$ (providing the flexibility) and the first part of the path $a$ (without flexibility).

As illustrated for the example above, Real Options valuation is the natural valuation technique for two-phased investment decisions, with an optional second phase (e.g. only performed if market situation is favourable). By the time of the second phase of 
the investment, the market situation is already more clear, so that a well-advised decision can be taken ${ }^{3}$.

\section{Case study: comparison of capacity leasing approaches for Belgian scale network}

We consider the three network deployment scenarios (IRU on DF, DF lease, lambda lease) discussed in Fig. 2, but assume now that the first five years of the planning horizon can be seen as a kind of learning time, over which some information considering the unknown evolutions in the rest of the planning horizon (the last 10 years in this example) becomes clear. We consider the case of Fig. 4, where one of the considered scenarios is chosen in year 0 and this scenario is followed during 5 years, after which we can choose to switch to another scenario. This means that year 5 is the only point of flexibility in this example (which is a simplification of the reality).

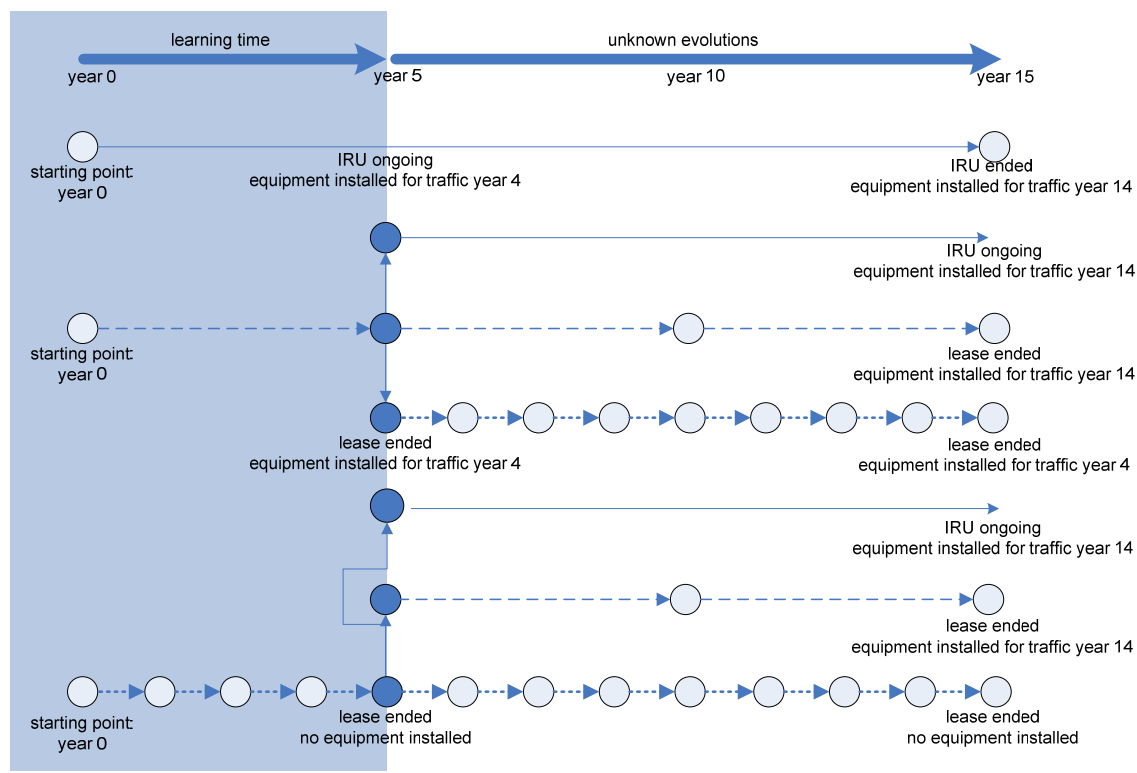

Fig. 4. Flexibility to react to changes at the end of the learning time

During the learning time of the first 5 years, a competitor might have entered the market, so that we can expect a drop in the market share in the coming years. Performing some customer surveys, it might even be possible to get an idea of the actual expected decrease of the market share and other implications. On the other hand, if a complementary service is becoming available, an increased market share

${ }^{3}$ Note that several option valuation techniques are distinguished in the literature. In this paper we only consider valuation through simulation, which is the most intuitive technique. 
can be estimated in a similar way. If we have started our network roll-out in year 0 using DF lease or lambda lease, the contract is ended and we can enroll in any new leasing contract of our choice.

We consider a network on the Belgian scale $(32,545 \mathrm{~km} 2)$ consisting of 3 interconnected rings with a total link length of $1488 \mathrm{~km}$. We consider a planning horizon of 15 years, with an expected annual traffic growth of $60 \%$. The reference traffic described in [2] was assumed to be obtained in the fourth year of the period studied. We assume OADMs in the nodes, with the costs described in Tab. 1. Equipment cost is expected to undergo a cost erosion of $10 \%$ a year. The costs and durations for the different considered network deployment scenarios are those of Fig. 1. The costs for dark fiber and wavelength lease are considered to be constant over time. This assumption originates from the observation that labour costs increase over time (contrary to equipment costs) and that in the future dark fiber might become scarcer.

We have calculated the cash-out flows for the three considered scenarios (IRU on DF, DF lease and lambda lease), i.e. the expenses for the leasing contracts as well as the required optical layer equipment. All network scenarios are dimensioned in order to cope with the expected traffic demand (so that the network operator's revenues or cash-in flows are equal for all scenarios). For lease contracts with durations of over one year (granularity of the traffic predictions), i.e. the dark fiber lease for 5 years and the IRU on dark fiber for 15 years in our study, the expected need of capacity is calculated at the beginning of the contract term in order to determine the required contract size. In case the traffic demand is rather static or well predictable, this approach leads to good results, as better rates can be negotiated in case the contract is taken for multiple fibers at the same time. When the actual demand exceeds the forecasts, additional wavelengths/fibers are leased or equipment is bought according to necessity.

We have considered an uncertain future traffic scenario, in order to simulate the fact that the actual traffic will be equal to the predicted traffic. In case the actual required line capacity is bigger than expected, the lease contract is immediately expanded and the additional required node capacity is bought and installed. In case of a traffic stagnation or decrease, leasing contracts are changed at the end of the term according to necessity, no new equipment is installed.

We consider the following uncertain traffic scenarios for the last 10 years of the planning horizon. The traffic is supposed to follow the forecasted traffic growth of $60 \%$ a year, except from an abrupt change in that trend in year 6 . We assume the information about this change to be known at the end of year 5 (e.g. competitor has entered the market). The abrupt change is modelled as a traffic increase or decrease somewhere between 0.01 times its current size and 10 times is current size and simulated using Monte Carlo simulation, performed by Crystal Ball [12]. A continuous variable with uniform distribution is assumed in the simulation.

The cost of the second phase of our planning horizon (last ten years of Fig. 4), calculating both with and without flexibility (using the formulae (3) and (2 respectively) is depicted in Fig. 5 for the cases where either DF lease or lambda lease is used for the first phase ${ }^{4}$. The difference between the bars without and with

\footnotetext{
${ }^{4}$ In case of IRU on DF in phase 1 , there is no flexibility anyhow.
} 
flexibility indicates the additional cost for the second phase in the absence of flexibility. As we assume the revenues for all scenarios to be equal, the difference in costs equals the difference in value (different sign). The cost difference therefore represents the option value. This illustrates that Real Options valuation (taking into account the value of the inherent options of the migration paths) is the natural way to evaluate the network deployment cost in this example.

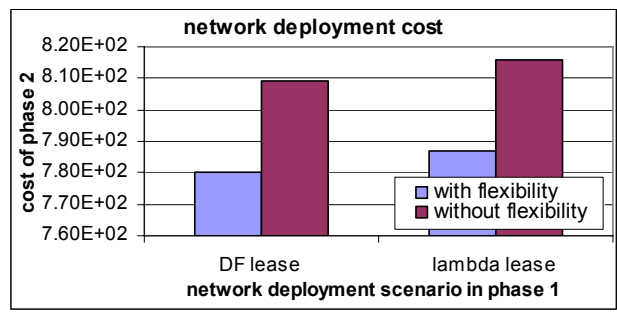

Fig. 5. Cost of second phase of network deployment

The real-life planning question to be answered in the considered case, is what network deployment scenario to start using in the beginning of the planning horizon (year 0 of our study). Traditionally, the evaluation will be made based on the expected traffic and cost evolutions, leading to the costs of the left bars in

Fig. 6. This evaluation shows that, in case everything evolves as expected, the dark fiber scenarios outperform lambda leasing in terms of costs.

However, when taking into account the uncertain evolutions in the second part of the planning horizon (abrupt change in year 6), the evaluation should consider the flexibility of some scenarios to easily react to those changes, by using formula (5). This means using the left bars of Fig. 5 (with flexibility) as the cost of the second phase, leading to the right bars in

Fig. 6 as the costs for the total planning horizon, which is exactly what Real Options evaluation suggests. This analysis shows the highest cost for the IRU on DF scenario, indicating that this is scenario is least flexible. The DF and lambda lease scenarios are more flexible. DF lease still comes out as the best solution.

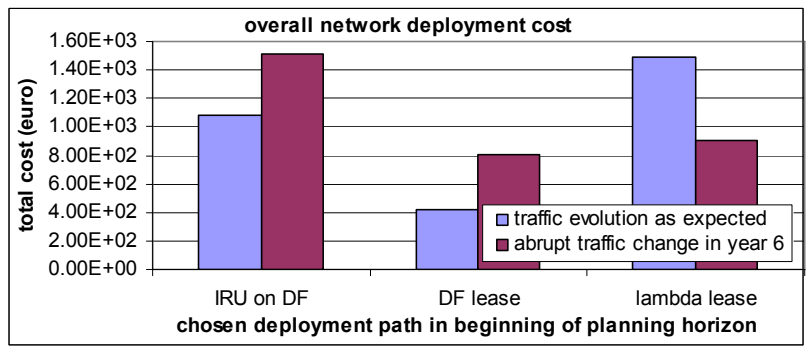

Fig. 6. Network deployment costs taken into account in the beginning of the planning horizon 


\section{Conclusions}

In this paper, we have compared different optical layer capacity leasing scenarios based on costs versus flexibility. Whereas an Indefeasible Right of Use on dark fiber is the cheapest per capacity unit per year and allows to cope with growing traffic without the need to update the contract immediately, the DF lease scenario was shown to offer the best trade-off between cost and flexibility as it allows to respond faster to decreasing traffic and it allows to switch sooner to another network scenario (IRU on DF or lambda lease). The Real Option valuation technique was shown to be the formalization of the natural way of evaluating the flexibility to switch between the scenarios, taking into account that information becoming available during the course of the planning horizon might influence the strategy followed.

Acknowledgments. We were supported by the EC through IST-Nobel2 and e1+, by IWT-Flanders through a grant for S.Verbrugge and by UGent through BOF-RODEO.

\section{References}

1. K. Maney, "Indefeasible rights of use is capturing nation", USA Today - Cyberspeak, February $27^{\text {th }} 2002$.

2. S. Verbrugge et al.: "Planning of transmission infrastructure to support next generation BELNET network," IST BroadBand Europe conference, Bordeaux, France, December 1214, 2005, papers online at www.bbeurope.org, p. W03B.02.

3. W. Van Dijk: "Acquisition and deployment of dark fiber within SURFnet", presented on CEF workshop, Prague, Czech Republic, May $25^{\text {th }}, 2004$.

4. A. Odlyzko: "Internet growth: myth and reality, use and abuse," Information Impacts Magazine, November 2000.

5. L. Lee: "An Introduction to Telecommunications Network Traffic Engineering”, Alta Telecom International Ltd., Edmonton, Alberta, Canada, 1986.

6. E. Jantsch: "Technological Forecasting in Perspective: A Framework for Technological Forecasting, its Techniques and Organisation", Organisation for Economic Co-operation and Development, available online..

7. S. Myers: "Determinants of Corporate Borrowing", Journal of Financial Economics, 1997, vol. 5, no. 2, pp.147-175.

8. Y. d'Halluin, P.A. Forsyth, K.R. Vetzal: "Managing Capacity for Telecommunications Networks under Uncertainty", IEEE/ACM Transaction on Networking, Aug. 2002, vol, 10 , pp. 579-588.

9. C. Kenyon, G. Cheliotis: "Dark fiber valuation", The Engineering Economist, 2002, vol. 47, no 3, pp. $264-308$.

10. W. De Maeseneire, "The real options approach to strategic capital budgeting and company valuation", Financiële Cahiers, LARCIER, 2006, ISBN: 2-8044-2318-2.

11. S. Verbrugge et al.: "Methodology and input availability parameters for calculating OpEx and CapEx costs for realistic network scenarios," Journal of Optical Networking, Feature Issue: Optical Network Availability, June 2006, vol. 5, no. 6, pp. 509-520.

12. Crystal Ball, http://www.decisioneering.com/ 